\title{
Tinha do couro cabeludo em crianças de Goiânia, Brasil
}

\author{
Tine capitis in children from G oiânia, Brazil \\ Tatiana Dias ${ }^{1}$, Orionalda de Fátima Lisboa Fernandes ${ }^{1}$, Ailton José Soares ${ }^{1}$, Xisto Sena Passos ${ }^{1}$, \\ Milce Costa ${ }^{1}$, Lúcia Kioko Hasimoto e Souza ${ }^{1}$ e Maria do Rosário Rodrigues Silva ${ }^{1}$
}

\begin{abstract}
Resumo Durante o período de janeiro de 1999 a julho de 2002 um total de 164 casos de tinha do couro cabeludo foram diagnosticados através de exames micológicos, realizados no Instituto de Patologia Tropical e Saúde Pública da Universidade Federal de Goiás. Destes pacientes, 94 (57,3\%) pertenciam ao sexo masculino, com idades variando de 3 meses a 13 anos. O diagnóstico e identificação dos agentes de dermatofitoses do couro cabeludo foram feitos utilizando-se exame direto com KOH a $20 \%$ e cultivo em ágar Mycobiotic e em ágar Sabouraud dextrose acrescido de cloranfenicol. As seguintes espécies foram identificadas: Microsporum canis $(71,3 \%)$, Trichophyton tonsurans (11\%), Trichophyton mentagrophytes $(7,9 \%)$, Trichophyton rubrum (6,7\%) and Microsporum gypseum (3\%). Nossos estudos mostraram que o fungo de habitat natural no animal (zoofílico), Microsporum canis foi o agente mais comum de lesões no couro cabeludo em humanos.
\end{abstract}

Palavras-chaves: Dermatófitos. Microsporum canis. Trichophyton tonsurans. Trichophyton mentagrophytes.

Abstract During the period January 1999 to July 2002 a total de 164 cases of Tinea capitis were diagnosed by mycological examination in Instituto de Patologia Tropical e Saúde Pública of the Universidade Federal de Goiás. Of the 164 patients 94 (57.3\%) were males, with an age of 3 months to 13 years. Laboratory studies were performed by direct examination with $20 \% \mathrm{KOH}$ and cultivated on Mycobiotic agar medium and Sabouraud dextrose agar with chloramphenicol. The following species were identified: Microsporum canis (71.3\%), Trichophyton tonsurans (11\%), Trichophyton mentagrophytes (7.9\%), Trichophyton rubrum (6.7\%), and Microsporum gypseum (3\%). Our study showed that the most frequent rise of scalp infection was a zoophylic fungi, called Microsporum canis.

Key-words: Dermatophytes. Microsporum canis. Trichophyton tonsurans. Trichophyton mentagrophytes.

As dermatofitoses são infecções fúngicas superficiais de tecidos queratinizados, causadas por um grupo de fungos que recebe a denominação de dermatófitos. Estes fungos são constituídos pelos gêneros Epidermophyton, Microsporum e Trichophyton que possuem características morfológicas, fisiológicas e antigênicas que permitem o seu relacionamento ${ }^{7}$. Os dermatófitos têm distribuição universal, entretanto a incidência das diferentes espécies e locais anatômicos da infecção pode ser influenciada por fatores geográficos, socio-econômicos e ambientais $^{1214}$.
As dermatofitoses podem invadir diferentes regiões do corpo, recebendo a denominação genérica de tinhas. A tinha do couro cabeludo é uma enfermidade que pode produzir lesões que variam desde a tonsura do pêlo até uma alopecia definitiva, sendo de maior ocorrência em crianças em idade escolar, constituindo-se um problema de saúde pública de grande importância no Brasil 69 .

Devido a importância epidemiológica desta micose, este trabalho teve como objetivo obter dados adequados de freqüência e etiologia de tinha do couro cabeludo, em Goiânia-GO, durante o período de janeiro de 1999 a julho de 2002.

1. Instituto de Patologia Tropical e Saúde Pública da Universidade Federal de Goiás, Goiânia, GO.

Endereço para correspondência: Prof ${ }^{a}$ Maria do Rosário Rodrigues Silva. IPTSP/UFG. R. Delenda Rezende de Melo, esq. com $1^{\text {a }}$ Avenida. Setor Universitário. Caixa Postal 131, 74605-050 Goiânia, GO

Tel: 62 209-6127, Fax: 62 521-1839

e-mail rosario@iptsp.ufg.br

Recebido para publicação em 24/9/2002

Aceito em 1/9/2003 


\section{MATERIAL E MÉTODOS}

Pacientes. Seis mil seiscentos e cinqüenta e dois (6.652) pacientes procedentes do Ambulatório de Dermatologia do Hospital das Clínicas da UFG, do Centro e Assistência Integrado de Saúde (CAIS) e de clínicas particulares de Goiânia foram enviados ao laboratório de Micologia do IPTSP da Universidade Federal de Goiás, para exame micológico. Destes, foram estudados 353 pacientes que possuíam lesões no couro cabeludo com suspeita clínica de dermatofitose.

Coleta, isolamento e identificação. O pêlo e as descamações do couro cabeludo coletados foram submetidos a exame microscópico direto com $\mathrm{KOH}$ a $20 \%$ e cultivados em meios de ágar Sabouraud dextrose contendo cloranfenicol (Becton Dickinson,
Cockeysville) e em ágar Mycobiotic (Difco, Detroit, Michigan) incubados a temperatura ambiente, por até 15 dias.

A identificação dos dermatófitos foi feita segundo metodologia de Rebell e Taplin, $1970^{13}$. De todos os isolados foram analisados as características macroscópicas como cor, superfície e reverso da colônia, e características microscópicas como a forma, tamanho e disposição dos conídios. Foi realizado ainda o microcultivo em lâmina, usando-se ágar batata para estimular a formação dos elementos de frutificação, permitindo uma melhor caracterização dos isolados. Teste da perfuração do pêlo in vitro e testes bioquímicos como a urease foram usados na diferenciação entre T. rubrum e T. mentagrophytes.

\section{RESULTADOS}

O exame microscópico direto com $\mathrm{KOH}$ das 353 amostras examinadas com lesões do couro cabeludo mostrou a presença de filamentos de dermatófitos em 164 (46,4\%).

Os pacientes com lesões do couro cabeludo por dermatófitos constituíram-se exclusivamente de crianças, sendo que ambos os sexos foram afetados em proporções semelhantes. Dos 164 pacientes, 144 $(87,7 \%)$ estavam entre 3 e 13 anos e $94(57,3 \%)$ pertenciam ao sexo masculino.
A identificação dos dermatófitos permitiu a caracterização de seis (6) espécies diferentes como agentes de tinha do couro cabeludo. Observou-se que entre as espécies isoladas, Microsporum canis foi o agente predominante, com 71,3\% (117/164) dos casos, seguido de Trichophyton tonsurans e de Trichophyton mentagrophytes com $11 \%(18 / 164)$ e $7,9 \%(13 / 164)$, respectivamente (Figura 1).

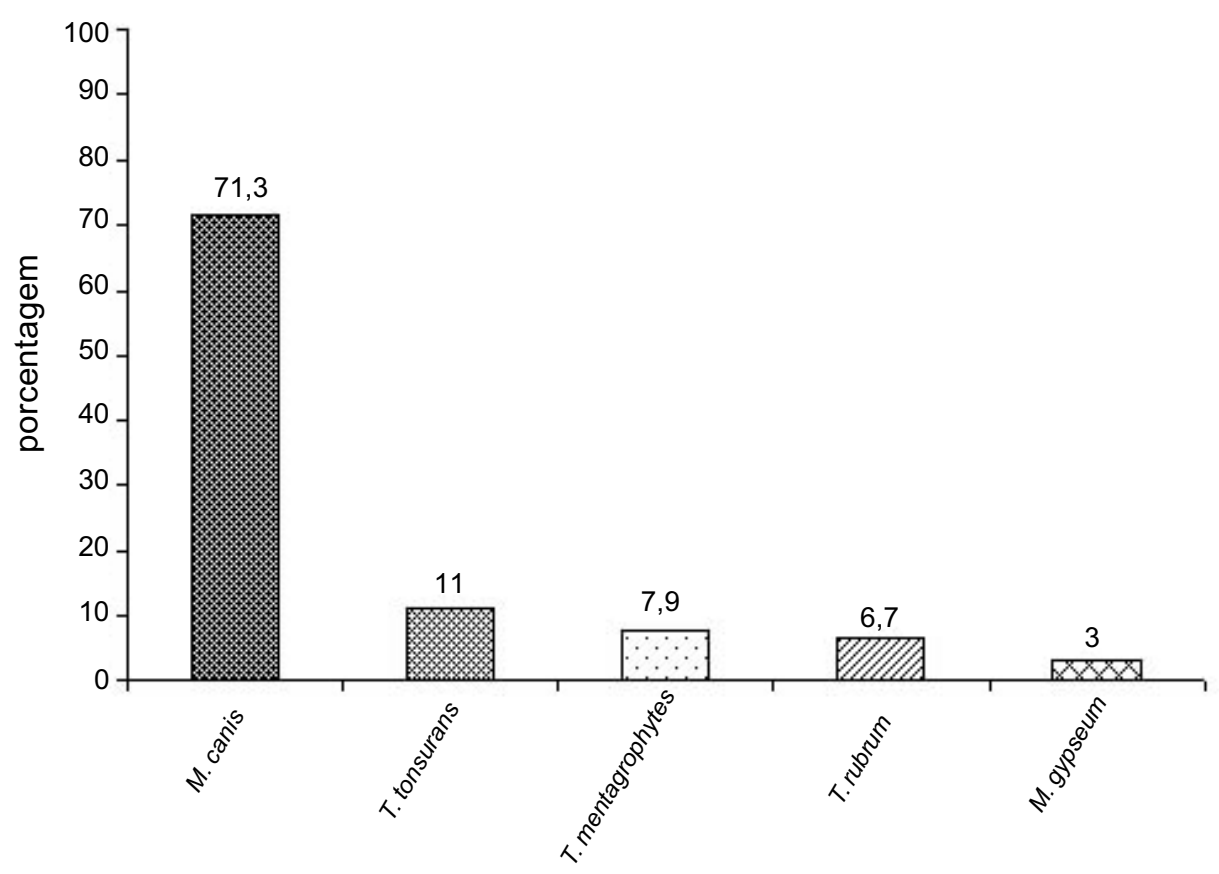

Figura 1- Distribuição por espécie de dermatófitos isolados de 164 pacientes com lesões no couro cabeludo. 


\section{DISCUSSÃO}

Embora tinea capitis seja uma das mais antigas infecções descritas no ser humano e da vasta experiência em seu tratamento clínico, ainda é hoje largamente distribuída pelo mundo, persistindo como um grave problema médico sanitário entre crianças na faixa etária escolar ${ }^{145}$. Nossas amostras de lesões do couro cabeludo foram caracterizadas por uma maior freqüência entre as crianças de 3 a 13 anos, com uma pequena predominância no sexo masculino. $O$ predomínio de dermatofitose do couro cabeludo nesta faixa de idade está relacionado com a ausência de ácidos graxos de cadeia média (C8-C12) que inibem o desenvolvimento de dermatófitos nesta região do hospedeiro ${ }^{8}$. Dados de Menan et $a^{11}$ associam tinha do couro cabeludo ao sexo masculino e às crianças na idade entre 8 e 11 anos.

As tinhas são afecções exógenas, cuja fonte de propagação está no homem ou animal doentes e, mais raramente, no solo. A prevalência de dermatófitos antropofílicos ou zoofílicos está intimamente relacionada a determinadas características regionais, levando-se em consideração fatores climáticos, graus de desenvolvimento social, representados pela urbanização e industrialização, os quais acarretariam mudanças nos hábitos de higiene pessoal 23101214 .

Baseando-se nos resultados encontrados no presente trabalho pode ser concluído que a fonte de infecção dos casos de lesões do couro cabeludo na nossa região é de origem zoofílica, visto que a maior porcentagem das lesões $(71,3 \%)$ foi causada por $M$. canis parasita de animais domésticos, o que explica provavelmente esta grande incidência em crianças que têm maior contato com gatos e cães. Afastar definitivamente os animais domésticos do homem, não representa uma forma adequada de evitar a propagação da doença, no entanto, o diagnóstico e tratamento adequados dos animais provavelmente serão de grande importância no controle desta infecção.

\section{REFERÊNCIAS BIBLIOGRÁFICAS}

1. Arenas R. Dermatofitosis en México. Revista Iberoamericana de Micologia 19:63-67, 2002.

2. Aste N, Pau M, Biggio P. Tinea capitis in adults. Mycoses 39:299301, 1996.

3. Costa M, Passos XS, Souza LKH, Miranda ATB, Lemos JA, Oliveira Júnior JO, Silva MRR. Epidemiologia e etiologia das dermatofitoses em Goiânia, GO, Brasil. Revista da Sociedade Brasileira de Medicina Tropical 35:19-22 2002.

4. Escutia B, Febrer I, Pemán J, Oliver V, Sánchez-Carazo J. Tinea capitis por Microsporum audouinii. Revista lberoamericana de Micologia 18:88-90, 2001.

5. Fernandes NC, Akiti T, Barreiros MGC. Dermatophytosis in children: study of 137 cases. Revista do Instituto de Medicina Tropical de São Paulo 43:83-85, 2001.

6. Furtado MSS, Ihára LT, Maróya MF. Tinea capitis na cidade de Manaus-AM. Anais Brasileiro de Dermatologia 60:315-318, 1985.

7. Gupta AK, Einarson TR, Summerbell RC, Shear NH. An overview of topical antifungal therapy in dermatomycoses. A North American perspective. Drugs 55: 645-674, 1998.

8. Hay RJ. Dermatoficias y outras micosis superficiales. In: Mandell GL, Gordon Douglas R, Bennett JE (eds) Enfermedades

Infecciosas. Principios y prática. Buenos Aires, Panamericana 3137-2149, 1991.

9. Matte SMW, Lopes JO, Melo IS, Beber AAC. A focus of favus due to Trichophyton schoenleinii in Rio Grande do Sul. Revista do Instituto de Medicina Tropical de São Paulo 39:1-3, 1997.

10. Mazón A, Salvo S, Vives R. Estudio etiológico y epidemiológico de las dermatofitosis em Navarra (España). Revista Iberoamericana de Micologia 14:65-68, 1997.

11. Menan El, Zongo-Bonou O, Rouet F, Kiki-Barro PC, Yavo W, N'Guessan FN, Kone M. Tinea capitis in schoolchildren from Ivory Coast (western Africa). A 1998-1999 cross-sectional study. International Journal of Dermatology 41:204-207, 2002.

12. Pereiro M, Pereiro E, Pereiro M Jr, Pereiro M. Incidencia de los dermatofitos em España desde 1926 a 1994. Actas DermoSifiliogr 87:77-84, 1996.

13. Rebell G, Taplin D. The dermatophytes. Their recognition and identification. University of Miami Press, Coral Gables, 1970.

14. Rubio MC, Rezusta A, Tomás JG, Ruesca RB. Perspectiva micológica de los dermatofitos en el ser humano. Revista Iberoamericana de Micologia 16:16-22, 1999. 